\title{
Nuevos registros de bivalvos marinos para el Perú
}

\section{New records of marine bivalves from Peru}

\author{
Carlos Paredes y Franz Cardoso
}

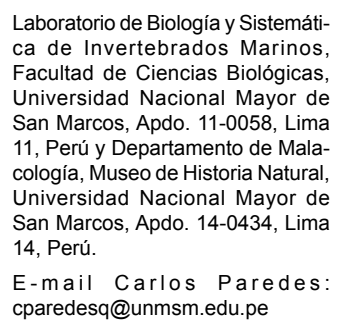

\section{Introducción}

Los moluscos son uno de los grupos de invertebrados más conocidos, sin embargo, continuamente información acerca de las variaciones en la distribución geográfica de las especies marinas es reportada. En el Perú, queda mucho trabajo al respecto, principalmente porque las colectas se realizan mayormente en la zona intermareal por razones de logística, lo cual es consecuencia del escaso apoyo económico que reciben las investigaciones para los inventarios de la biodiversidad marina, no obstante su importancia. Los autores venimos trabajando desde hace varios años en la elaboración de un "Catálogo de moluscos bivalvos de la costa peruana”, habiendo tenido escasas oportunidades de realizar colectas en los niveles infralitorales del mar.

Respecto a los Bivalvia Protobranchia, que viven en la zona infralitoral, se reporta por primera vez para el mar peruano, dos especies que se suman a las registradas anteriormente por los autores (Paredes y Cardoso, 2001).

Dentro de las cuatro especies de la subclase Pteriomorphia que se registran por primera vez para el Perú, tres son intermareales, y una vive en el sublitoral.

\section{Material y métodos}

La colecta del material se realizó a mano, en la zona intermareal y mediante dragado o buceo autónomo en el infralitoral, durante los trabajos de campo programados en nuestras actividades de investigación sobre la diversidad de la malacofauna peruana. Malletia goniura fue colectada durante el Crucero BIC HUMBOLDT 9607-08 (Kameya et al., 1997), Delectopecten zacae durante el Crucero 7911-12 Profesor Siedlecki, y Adrana sowerbyana dentro de la fauna acompañante del "caballito de mar", Proyecto del Instituto del Mar del Perú.

El material fue fijado con formol al 7\% neutralizado con bórax y conservado en alcohol etílico al 70\%. Para la determinación taxonómica se utilizó la bibliografía especializada y se contó con el apoyo del doctor Eugene Coan y Paul ValentichScott, curadores del Museo de Historia Natural de Santa Barbara (SBMNH). Para el ordenamiento sistemático se siguió a Coan et al. (2000). El material está depositado en las colecciones del Museo de Historia Natural de la Universidad Nacional Mayor de
San Marcos (MUSM) y el Laboratorio de Biología y Sistemática de Invertebrados Marinos de la Facultad de Ciencias Biológicas de la Universidad Nacional Mayor de San Marcos (LaBSIM).

\section{Sistemática}

\section{Subclase Protobranchia}

Orden NuCULOIDA

Superfamilia NuculanoIdeA H. Adams \& A. Adams, 1858

Familia Nuculanidae H. Adams \& A. Adams, 1858

Subfamilia Nuculaninae H. Adams \& A. Adams, 1858

Genero Adrana H. Adams \& A. Adams, 1858

\section{Adrana sowerbyana (Orbigny, 1845)}

(Figura 1)

Leda sowerbyana Orbigny, 1845: 544.

Yoldia (Adrana) sowerbyana, Dall, 1909: 251.

Adrana sowerbyana, Hertlein \& Strong, 1940: 410-411; Olsson, 1961: 69-70, pl. 3, figs. 3, 3a; Keen, 1971: 33, fig. 41; Bernard, 1983: 11.

Concha alargada y comprimida, con el extremo anterior redondeado y posterior truncado; picos pequeńos y deprimidos, situados delante de la línea media, aproximadamente a tres cuartas partes de la distancia entre ésta y el extremo anterior; borde ventral convexo, ligeramente sinuado cerca de los extremos; margen dorsal casi recto, con el extremo posterior ligeramente dirigido hacia arriba; lúnula y escudo alargados y estrechos, ligamento, dientes y seno paleal como en el género. Escultura con finas líneas concéntricas, que son más finas en la parte ventral posterior y más gruesa en la región anterior, donde siguen la sinuosidad del margen ventral. Color externo amarillento grisáceo, blanquecino en la región umbonal, y con dos rayos lisos más claros debajo del margen dorsal posterior; interior blanquecino aporcelanado. Longitud, 41,5 mm.

Distribución: Panamá a Ecuador (Keen, 1971).

Hábitat: Fondo areno-fangoso, infralitoral.

Nueva localidad: Piura (Máncora).

Material examinado: 1 lote, 1 ejemplar (LaBSIM).

Observaciones: Esta especie fue determinada por Paul Valentich-Scott. 


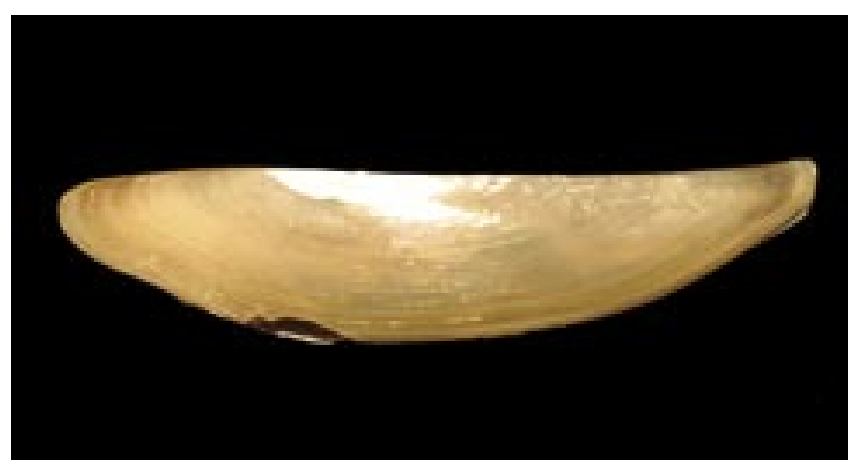

Figura 1. Adrana sowerbyana, longitud $41,5 \mathrm{~mm}$.

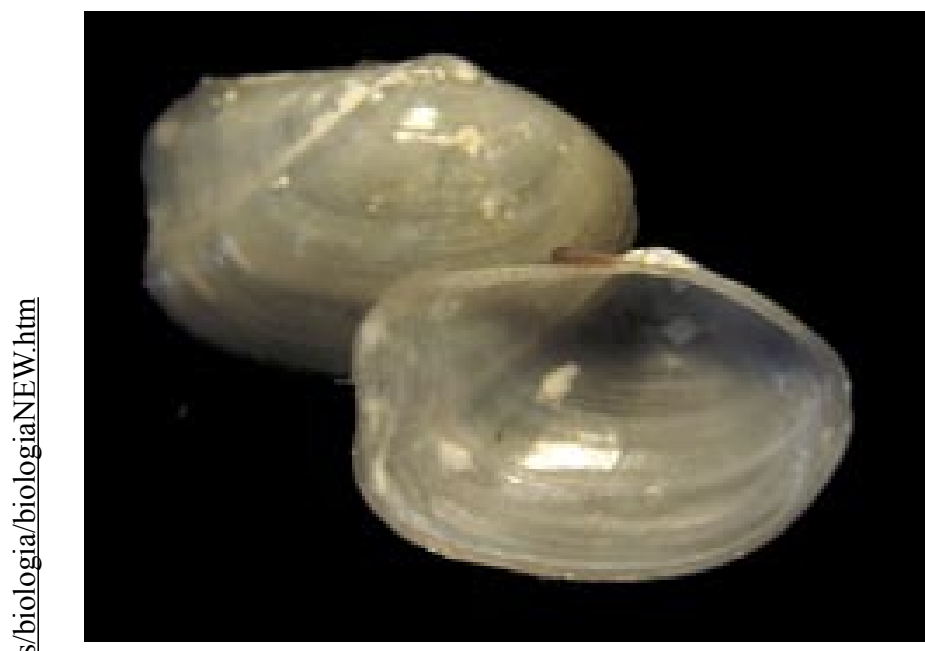

Figura 2. Malletia goniura, longitud $14,8 \mathrm{~mm}$.

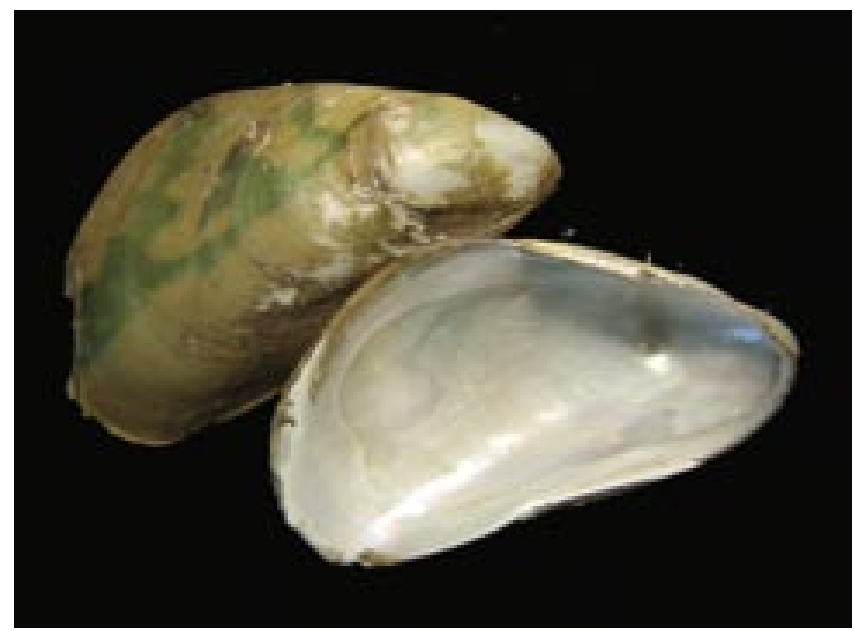

Figura 3. Mytilus strigatus, longitud $19,3 \mathrm{~mm}$.

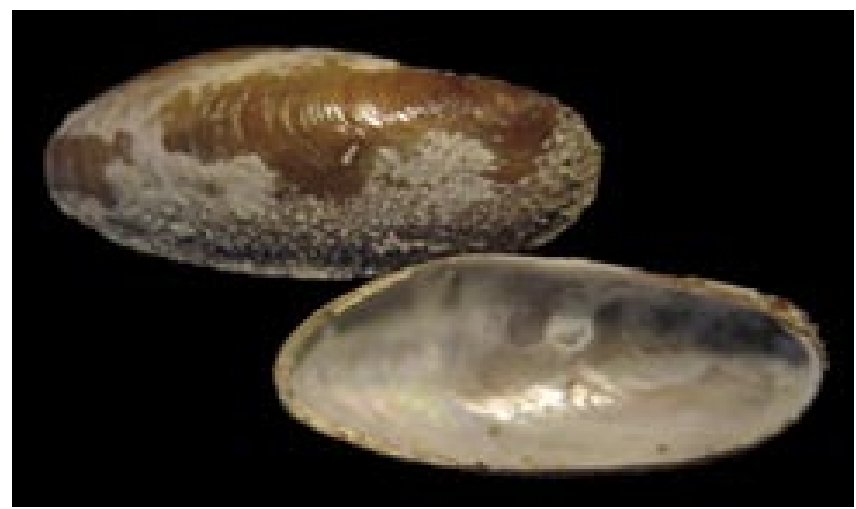

Figura 4. Leiosolenus spatiosa, longitud $8,2 \mathrm{~mm}$.

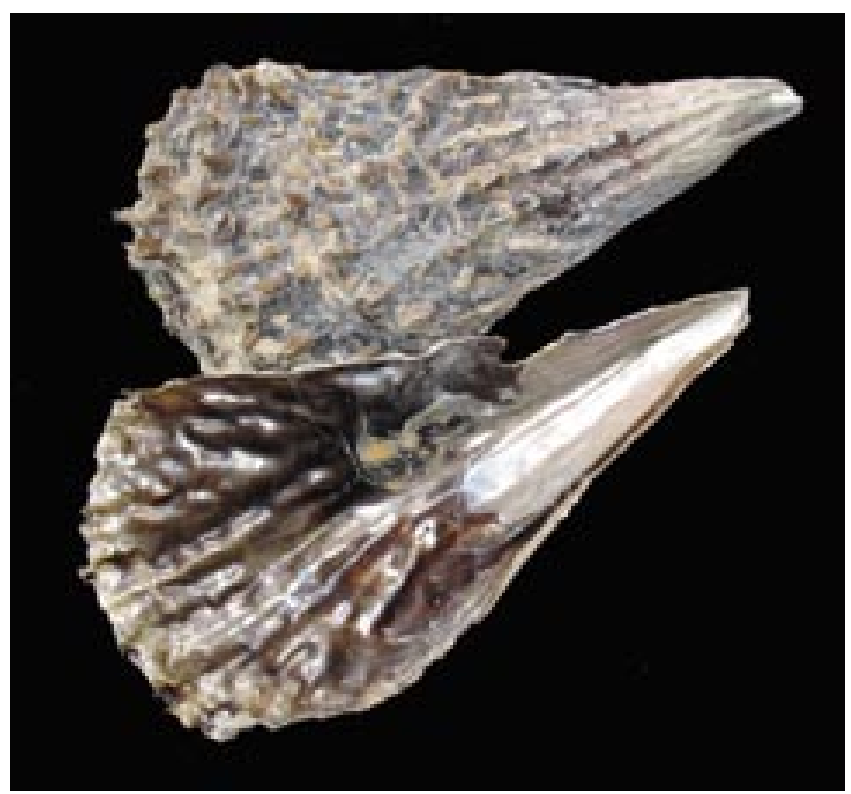

Figura 5. Pinna rugosa, longitud $290,3 \mathrm{~mm}$.

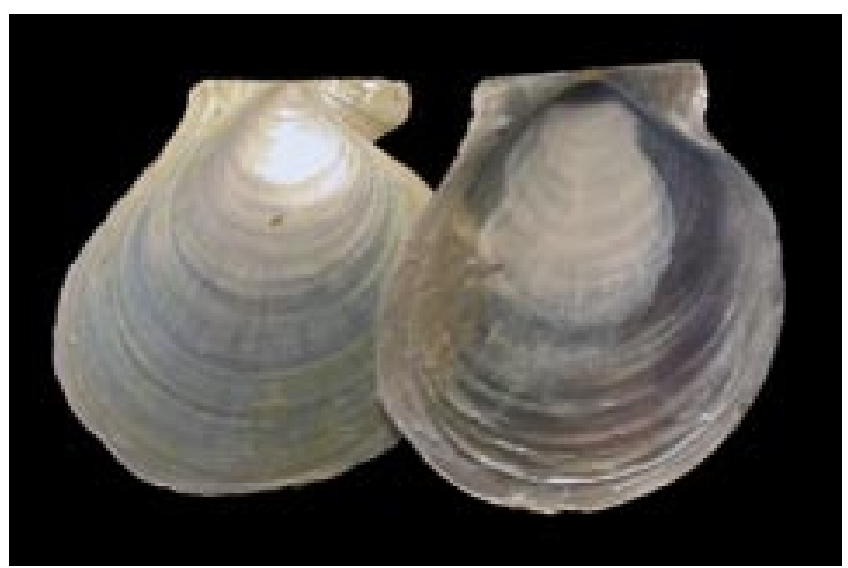

Figura 6. Delectopecten zacae, longitud $25,8 \mathrm{~mm}$.

Familia Malletindae H. Adams \& A. Adams, 1858

Genero MaLLETIA des Moulins, 1832

\section{Malletia goniura Dall, 1890}

(Figura 2)

Malletia goniura Dall, 1890: 251, pl. 10, fig. 10. Bernard, 1983: 14. Malletia (Neilo) goniura, Hertlein \& Strong, 1940: 422; Keen, 1971: 35, fig. 49.

Malletia (Malletia) goniura, Bernard, 1983: 10.

Concha oval frágil subnacarada, algo inflada en la región umbonal, con dos lomos radiales que se proyectan desde el pico hasta el borde posterior truncado; charnela con ligamento opistodético, largo y prominente, los dientes son aguzados, 17 a 19 anteriores y 25 a 27 posteriores; el seno paleal es profundo. Escultura con finas líneas con marginales. Color exterior pardo amarillento, con periostraco pulido y brillante, interior blanquecino translúcido. Longitud, 14,8 mm.

Distribución: Panamá a Ecuador (Keen, 1971).

Nueva localidad: Piura $\left(05^{\circ} 08^{\prime} \mathrm{S}, 81^{\circ} 29^{\prime} \mathrm{W}\right)$.

Hábitat: Fondo fangoso, $852 \mathrm{~m}$.

Material examinado: 1 lote, 6 ejemplares (LaBSIM, MUSM). 
Observaciones: Comisuras anterior y posterior presentes en todos los ejemplares. Según Bernard (1983) esta especie vive en aguas profundas (entre 1350 y 3350 metros).

\author{
Subclase PTERIOMORPHIA \\ Orden MytiloIDA \\ Superfamilia Mytiloidea Rafinesque, 1815 \\ Familia Mrtilidae Rafinesque, 1815 \\ Subfamilia Mytilinae Rafinesque, 1815 \\ Genero MYtiLus Linnaeus, 1758
}

\section{Mytilus strigatus Hanley, 1843}

(Figura 3)

Mytilus strigata Hanley, 1843: 251, ex Hinds MS; 1856: 388; pl. 24. Modiolus arciformis, Dall, 1909: 258.

Volsella (Volsella) arciformis, Hertlein \& Strong, 1946: 72, pl. 1, fig. 5 .

Mytella falcata, Soot-Ryen, 1955: 51-52, pl. 5, fig. 24, text-figs. 35, 38-40, 45.

Mytilus arciformis, Olsson, 1961: 113-114, pl. 12, figs. 4, 4b.

Mytella arciformis, Keen, 1971: 63, fig. 124.

Mytella strigata, Keen, 1971: 63, fig. 127; Bernard, 1983: 18; Cruz, 1986: 135-136; Cruz \& Jimenez, 1994: 27, fig. 8;Skoglund, 2001: 17

Concha alargada, estrecha y arqueada, con el borde ventral comprimido, lo cual origina un fuerte lomo umbonal, el pico es terminal y el borde dorsal más arqueado hacia la parte posterior; charnela con dos o tres dientes débiles; el resilio es casi tan largo como el margen dorsal posterior. Escultura con finas líneas concéntricas, claramente distinguibles de las de crecimiento. Coloración exterior pardo olivácea con manchas negruzcas transversales, más amplias en la región posterior; el periostraco es delgado y liso; color interior purpúreo y nacarado. Longitud, 19,3 mm.

Distribución: Guaymas, Sonora, México (Keen, 1971) a Golfo de Guayaquil e Isla Galápagos, Ecuador (Olsson, 1961).

Nueva localidad: Tumbes (Cancas).

Hábitat: Fondo blando.

Material examinado: 1 lote, 4 ejemplares (LaBSIM).

Subfamilia Lithophaginae H. Adams \& A. Adams, 1857 Genero LEIosolenus Carpenter, 1857

\section{Leiosolenus spatiosa (Carpenter, 1857)}

(Figura 4)

Leiosolenus spatiosa Carpenter, 1857: 130.

Lithophaga (Leiosolenus) spatiosa, Soot-Ryen, 1955: pl. 10, fig. 59; Olsson, 1961: 136; Bernard, 1983: 21; Keen, 1971: 70, fig. 142, 142a; Gemmell et al., 1987: 23.

Leiosolenus spatiosa, Skoglund, 2001: 21.

Concha delgada casi cilíndrica alargada, con los extremos redondeados y los picos pequeńos subterminales; margen dorsal recto hasta donde se inicia, aproximadamente, el tercio posterior, luego baja hasta el extremo; margen ventral ligeramente curvado. Superficie cubierta por incrustaciones calcáreas, que en la parte anterior y ventrolateral de la valva, desarrollan como arrugas irregulares verticales, y no se proyectan más allá del extremo posterior. Donde no hay incrustaciones se nota el periostraco de color pardo y se aprecian las líneas concéntricas de crecimiento; el interior es blanquecino y brillantemente nacarado. Longitud, 8,2 $\mathrm{mm}$.

Distribución: San Felipe, Golfo de California, a Ecuador (Keen, 1971).

Nueva localidad: Tumbes (Bocapán).

Hábitat: Intermareal rocoso, sobre Crassostrea columbiensis. Material examinado: 1 lote, 2 ejemplares (LaBSIM).

\author{
Orden Pterioida \\ Suborden Pinnina \\ Superfamilia Pinnoidea Leach, 1819 \\ Familia PinNidae Leach, 1819 \\ Genero PINNa Linnaeus, 1758
}

Pinna rugosa G.B. Sowerby I, 1835

(Figura 5)

Pinna rugosa G.B. Sowerby I, 1835: 84; Hertlein \& Strong, 1943: 165; Olsson, 1961: 143-144, pl. 18, fig. 1; Abbott \& Dance, 1982: 300; Bernard, 1983: 22; Cruz \& Jiménez, 1994: 30, fig. 11; Skoglund, 2001: 23.

Concha alargada subtriangular, con el borde posterior redondeado y el pico terminal; profundamente excavada en su mitad anterior y expandida en la posterior; escultura con 7 hileras de espinas tubulares oblicuas, más desarrolladas en la parte media y posterior, en esta última, se intercalan cuatro hileras de espinas más cortas que se inician en el tercio posterior; no hay espinas en la parte anterior, pero se presentan cuatro costillas radiales que siguen la dirección de las hileras de espinas, y además se presenta una quilla más oscura. Exterior color café ámbar, con sombras negruzcas; el color interior es similar al exterior, pero está brillantemente nacarado, siendo la capa de nácar más gruesa en la mitad anterior de la concha, la que tiene color blanco perlado y está dividida en dos lóbulos por un surco longitudinal, el cual origina la quilla externa. Longitud, 290,3 mm.

Distribución: Cerca de la cabeza del Golfo de California en San Felipe, Baja California, México (Gemmell et al., 1987), a Salinas, Ecuador, e Islas Clipperton (Salvat \& Salvat, 1972).

Nueva localidad: Piura (Bahía de Sechura).

Hábitat: Intermareal, fondo areno-fangoso.

Material examinado: 1 lote, 1 ejemplar (LaBSIM).

\author{
Orden Ostreoida \\ Suborden Pectinina \\ Superfamilia Pectinoidea Rafinesque, 1815 \\ Familia Pectinidae Rafinesque, 1815 \\ Subfamilia Captonectinae Habe, 1977 \\ Genero DeLeCtopecten Stewart, 1930
}

\section{Delectopecten zacae (Hertlein, 1935)}

$$
\text { (Figura 6) }
$$

Pecten (Delectopecten) zacae Hertlein, 1935: 321. Delectopecten zacae, Keen, 1971: 91, fig. 194; Skoglund, 2001: 29. Cyclopecten zacae, Bernard, 1983: 26.

Concha delgada, típica de la familia por su forma, valvas delgadas poco convexas; la aurícula posterior está integrada al disco, y la aurícula anterior derecha con la muesca bisal provista de ctenolium (hilera de dientes pequeños); escultura consiste en 
finas estrías radiales y concéntricas con pequeñas pústulas en las intersecciones; en la parte anterior de la valva derecha hay 5 a 8 costillas radiales escamosas, todas se extienden desde el pico y alcanzan el borde ventral; la aurícula derecha esculturada con costillas concéntricas, más fuertes en el triángulo distal que en el proximal, el cual es ligeramente deprimido; las costillas del triángulo distal presentan nódulos dispuestos de tal manera que forman 4 costillas radiales que se inician en el pico, y 2 más o menos en la parte media. Color exterior blanquecino rosáceo iridiscente; color interior brillantemente nacarado, aporcelanado en la región umbonal. Longitud, 25,8 mm.

Distribución: Rocas Alijos, México (McLean \& Coan, 1996) a Panamá; incluyendo Clipperton y las Islas Galápagos (Keen, 1971).

Nueva localidad: Lambayeque $\left(06^{\circ} 40,2^{\prime} \mathrm{S}, 80^{\circ} 44,6^{\prime} \mathrm{W}\right)$.

Hábitat: Fondo blando, $140 \mathrm{~m}$.

Material examinado: 1 lote, 6 ejemplares (LaBSIM, MUSM).

\section{Discusión}

Hasta el trabajo de Paredes \& Cardoso (2001) se habían reportado 23 especies de protobranquios para el mar peruano. Posteriormente fue registrada Nucula (Nucula) pisum, especie de aguas frías (Cornejo \& Paredes, 2004), cuyo límite norte de distribución era la Bahía de Mejillones en Antofagasta, Chile (Villarroel \& Stuardo, 1998). Si añadimos las dos especies de aguas ecuatoriales, registrados en el presente trabajo, se incrementa a 26 el número de bivalvos Protobranchia conocidos para la costa peruana.

De otro lado, cabe destacar que dentro del género Adrana, solamente se había registrado Adrana crenifera, por lo que $A$. sowerbyana, es la segunda especie de este género, hallada en nuestro país; lo mismo ocurre con Malletia goniura, ya que antes sólo era conocida en aguas peruanas, $M$. peruviana.

También llama la atención, el hallazgo de $M$. goniura, a solo 800 metros de profundidad, si se tiene en cuenta que esta especie tenía un rango de profundidad entre los 1350 y 3050 metros (Bernard, 1983).

Finalmente, en todos los casos, las especies registradas amplían su distribución latitudinal hacia el sur de la zona ecuatorial, y cinco han sido halladas en la zona de transición de la Provincia Panameña, entre los Departamentos de Tumbes y Piura, sin llegar a rebasar la llamada "zona buffer" de Paita (Vegas-Vélez, 1980). Diferente es el caso de Delectopecten zacae, especie conocida, por el sur, hasta Panamá (Keen, 1971), y colectada en 1979 en Lambayeque, dentro del ámbito de la Provincia Peruana.

\section{Literatura citada}

Abbott, R.T. \& S.P. Dance. 1982. Compendium of Seashells. E. P. Dutton, Inc. New York. 410 pp.

Bernard, F.R.1983. Catalogue of the living bivalvia of the Eastern Pacific Ocean: Bering Strait to Cape Horn. Canadian Special Publication of Fisheries and Aquatic Sciences 61: $102 \mathrm{pp}$.

Coan, E.V., P. Scott \& F. R. Bernard. 2000. Bivalve Seashells of Western North America: Marine Bivalve Mollusks from Artic Alaska to Baja California. Santa Barbara Museum of Natural History Monographs Number 2: 764 .

Cornejo, O. \& C. Paredes. 2004. Nuevos bivalvos para el Perú en Bahía Independencia. Rev. peru. biol. 11(1): 41-44.

Cruz, M. 1986. Contribución al conocimiento de los bivalvos vivos en los Esteros de El Salado y Cascajal del Golfo de Guayaquil interior. Pub. INOCAR, Ecuador 3(1): 133-148.

Cruz, R.A. \& J.A. Jimenez. 1994. Moluscos asociados a las áreas de manglar de la Costa Pacífica de América Central: Guia. Heredia, C.R.: EFUNA. 182 pp.

Dall, W.H. 1909. Report on a collection of shells from Perú, whith a summary of the littoral marine Mollusca of the Peruvian Zoological Province. Proc. U.S.N. Mus. 37(1704): 147-294.

Gemmell, J., B.W. Myers \& C.M. Hertz. 1987. A faunal study of the bivalves of San Felipe and environs, Gulf of California, from the Gemmell Collection (1965 to 1976). Festivus 18(Supplement): 1-72.

Hertlein, L.G. \& A.M. Strong. 1940-1946. Eastern Pacific Expeditions of the New York Zoological Society. Mollusks from the West Coast of Mexico and America Central. Parts I-III. Zoologica, New York Zoological Society, vols. 25-31.

Keen, A.M. 1971. Sea shells of tropical West America. 2a. ed. Stanford Univ. Press, California, 1064 pp., 3325 figs. 22 pls.

Kameya, A., R. Castillo, L. Escudero, E. Tello, V. Blaskovic, J. Cordova, Y. Hooker, M. Gutierrez \& S. Mayor. 1997. Localización, distribución y concentración de langostinos rojos de profundidad. Crucero BIC Humboldt 9607-08 (18 de julio a 06 de agosto de 1996). Pub. Esp. Inst. Mar Perú: 1-47.

McLean, J.H. \& E.V. Coan. 1996. Marine Mollusks of Rocas Alijos, pp. 305-318. In Rocas Alijos Scientific Results from the Cordell Expeditions. Ed. Robert W. Schmieder. Kluwer Academic Publishers. Boston $\mathrm{xxx}+481 \mathrm{pp}$.

Paredes, C. \& F. Cardoso. 2001. Nuevos registros de Protobranchia (Mollusca: Bivalvia) para el mar peruano. Rev. peru. biol. 8(1): 5-10.

Olsson, A.A. 1961. Mollusks of the tropical eastern Pacific. Paleonto. Res. Ins. Ithaca, New york. 574 pp., pls 1-86.

Salvat, B. F. Salvat. 1972. Geographic distribution of Pinna rugosa Sowerby, 1835 (Mollusca: Bivalvia). The Veliger 15(1): 43-44.

Skoglund, C. 2001. Panamic Province Molluscan literature. Additions and changes from 1971 through 2000. I. Bivalvia. The Festivus 32 (Suppl.): 1-119.

Soot-Ryen, T. 1955. A report on the family Mytilidae. Allan Hancock Pacific Exped. Univ. So. California Press, Los Angeles 20(1): 1-175, pls. 1-10, text figs. 1-78.

Vegas-Velez, M. 1980. Algunas consideraciones biogeográficas sobre el Pacífico Sudoriental. Bo. Inst. Oceanog., S. Paulo 29(2): 371-373.

Villarroel, M. \& J. Stuardo. 1998. Protobranchia (Mollusca: Bivalvia) chilenos recientes y algunos fósiles. Malacologia 40 (1-2): 113-229. 